\title{
EL AULA INCLUSIVA COMO RESPUESTA AUTOPOIÉTICA Y EL PERSONALISMO
}

\section{THE INCLUSIVE CLASSROOM AS AN AUTOPOITICAL RESPONSE ANID PERSONALISM}

\section{María Eugenia Picco ${ }^{1}$}

meugepicco@gmail.com

5493521511231

Universidad Nacional de Villa María

Villa María

Argentina

\section{RESUMEN}

El presente trabajo aborda la cuestión de la educación inclusiva en relación a la explicación que ofrece Niklas Luhmann desde su teoría de los sistemas. Para esto se partirá desde una aproximación a la caracterización de los principios fundamentales de la teoría de este autor. A la luz de esta posición, y desde la perspectiva del personalismo, se analiza el impacto de esta teoría en la estructuración y organización del sistema educativo en su modalidad inclusiva, considerándola como una

respuesta autopoiética del mismo sistema de modalidad común.

\section{PALABRAS CLAVE}

Binariedad, exclusión, personalismo, máquinas triviales, respuesta autopoiética, sistema complejo

\section{ABSTRACT}

This paper deals with the question of inclusive education in relation to the explanation offered by Niklas Luhmann from his theory of systems. In order to do this, the paper will start from an approximation to the characterization of the 
fundamental principles of this author's theory. In the light of this position, and from the perspective of Personalism, the impact of this theory on the structuring and organization of the educational system in its inclusive model is analyzed, considering it as an autopoietic response of the common education model system.

\section{KEYWORDS}

Autopoietic response, binarius, complex system, exclusion, personalism, trivial machines

\section{DESARROLLO}

\section{a) APROXIMACIÓN A LA TEORÍA LUHMANNIANA}

Niklas Luhmann fue un polémico sociólogo alemán del siglo XX que, de la mano de Talcott Parson y el estructural funcionalismo - aunque no coincidió plenamente con él -, desarrolló los fundamentos de su teoría de la sociedad desde una perspectiva abstracta, "estrictamente descriptiva y con pretensión de teoría omnicomprensiva de lo social" (Suárez, s/f) que lo llevó a mantener acaloradas discusiones con algunos académicos de la época tal como Jürgen Habermas.

Luhmann se interesó por la sociología a partir de 1960 cuando fue becado por la Universidad de Harvard después de haber estudiado derecho. Al haberse desempeñado como funcionario público antes de su partida a EE.UU. la burocracia y otros fenómenos al interior de la administración pública fueron inspiradores de sus intereses para el desarrollo de sus posteriores investigaciones y las formulaciones de sus tesis.

Según su teoría, Luhmann afirma que la sociedad puede explicarse en tanto se entienda como un sistema complejo, para lo que se hace necesario abandonar la tradicional postura sociológica en la que el hombre es el centro de las construcciones sociales y reemplazarlo por las comunicaciones que este produce al relacionarse con los elementos internos y externos del sistema. Es por esto que se podría considerar que su idea es tan provocativa como lo ha sido la de Copérnico al cambiar el modelo geocéntrico por el heliocéntrico.

Los cambios que Luhmann sugirió para fundamentar su teoría se puede observar en los gráficos a continuación:

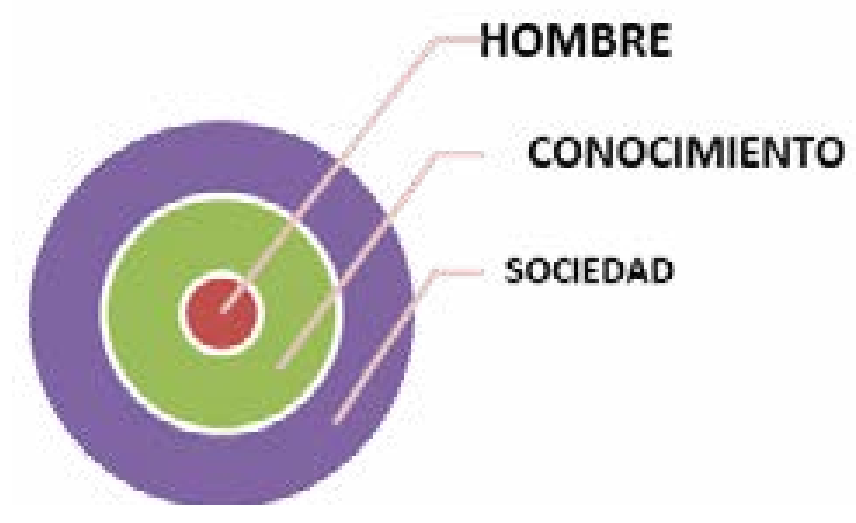

Figura 1: Fuente: Elaboración propia Concepción sociológica tradicional.

COMUNICACIÓN

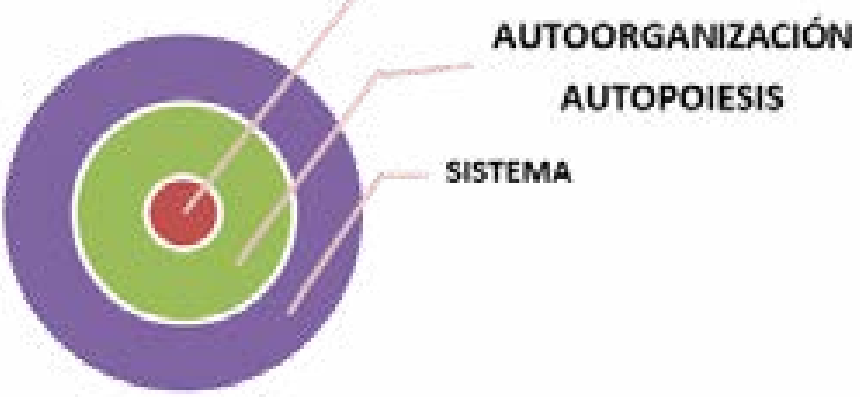

Figura 2: Fuente: Elaboración propia Concepción sociológica luhmanniana.

Tal puede apreciarse, en la Figura 1 se grafica la concepción sociológica en la cual el hombre se ubica en el centro y se encuentra mediado por el conocimiento en la conformación de la sociedad. Sobre esta no se ahondará ya que pertenece a la visión sociológica tradicional de occidente.

Aunque para este caso es importante destacar que a partir de la teoría de Talcott Parson - vinculada al funcionalismo de esta visión 
tradicional -, Luhman considera de manera crítica que este es el punto de partida para desarrollar un enfoque que coincida con los descubrimientos que a mediados del siglo anterior se sucedían en los ámbitos biológico y tecnológico, por lo que también toma el concepto desarrollado por los biólogos Humberto Maturana y Francisco Varela sobre autopoiesis para explicar el funcionamiento de un sistema, y que a partir del cual Luhmann identifica tres tipos de sistemas: el biológico, el psíquico y el social y los distingue a partir de que "cada uno tiene su modo de operación y reproducción" (García, s/f).

Desde aquí se aparta de Parson al señalar que la teoría de este es insuficiente para explicar los fenómenos que ocurren en la sociedad ya que no considera principalmente las contingencias que pueden ocurrir en las relaciones internas del sistema y también con su entorno. Según Luhman esto impide considerar "las otras posibilidades, en los equivalentes funcionales (punto de vista cambiable) que podrían ofrecer soluciones comparables a un mismo problema." (García, s/f). Siendo entonces que Luhmann toma en cuenta las contingencias, a este punto, se constituye en importante la selección entre las posibilidades existentes, de lo que se presume también que en la selección contingente de posibilidades deben ser tenidos en cuenta los riesgos que esta operación de seleccionar trae aparejada.

Así, en la Figura 2 aparece la propuesta de Luhmann en la cual la sociedad es un sistema complejo determinado en base a las relaciones y vínculos que se establecen hacia el interior y el exterior al que llama entorno, donde este sistema surge como necesidad para reducir la complejidad y por lo que, de acuerdo a su perspectiva, es posible entender que el entorno que se encuentra fuera del sistema posee menos organización que el interior del mismo a partir de la noción de clausura de operación de un sistema complejo en la que "el sistema depende totalmente de su propia organización" (García, s/f).

Ahora bien, "sin un cambio de actitud cognitiva es imposible comprender la teoría de Niklas Luhmann" (García, s/f) ya que es preciso no tan sólo considerar el cambio de perspectiva con respecto a lo que Luhmann identifica como núcleo del sistema social, sino que además es necesario tener en cuenta que para este sociólogo, un sistema se distingue de su entorno en base a las relaciones de diferencia que se presentan entre estos dos.

De esta diferenciación surge la autorreferencia, lo que le permite al sistema operar "empleando esta diferencia para obtener información" (García, s/f) y de esta forma establecer su estructura, la cual nunca será estática sino que atenderá a las transformaciones que se den en base a los vínculos que establezca el mismo sistema a partir de sus necesidades.

Por esto y según la teoría de los sistemas de Niklas Luhmann se puede señalar que los sistemas se caracterizan porque a partir de la noción de clausura de operación "las estructuras propias se pueden construir y transformar únicamente mediante operaciones que surgen en el mismo sistema" (García, s/f), entendiendo que estas operaciones son las comunicaciones que suceden en el sistema, lo que permite "conformar dentro del sistema dos acontecimientos fundamentales: la autoorganización y la autopoiesis" (García, s/f), de manera tal que estos procesos originan los elementos de los que se componen los sistemas. A su vez, los define como autopoiéticos ya que "producen todo lo que emplean como unidad a través de aquello que emplean como unidad" (García, s/f), es decir que tienen capacidad para reproducirse y mantenerse por sí mismos de acuerdo a las necesidades que surjan en su interior y son también autorreferenciales en tanto que "las ideas de autorreferencia deben 
ser trasladas desde los modelos de asimetría: reflejo, acto, intención, jerarquía, a modelos de simetría" (García, s/f). De esto que se entiende que para operar de manera autorreferencial es necesario la consideración de la diferencia, lo que permitirá la comprensión de la sociedad como sistema, siendo esta "una forma de observación y eventualmente una forma de descripción" (García, s/f) distinguiéndose de los sistemas que solo pueden ser comprendidos como los biológicos.

De esta manera Luhmann considera que la complejidad de un sistema no es un obstáculo para comprenderla, sino que es a partir de esta característica que el sistema se constituye, por lo que su teoría debe ser considerada como un instrumento para analizar la funcionalidad del sistema, que surge a partir de la necesidad de reducir la complejidad al interior del mismo, creando así subsistemas, los cuales poseerán entornos dentro del sistema, y nuevas relaciones que le permitan establecer vínculos entre el subsistema y el entorno del sistema para superar la complejidad del entorno, de este modo y según esta perspectiva, los sistemas logran evolucionar.

Ahora bien, de la comparación entre los gráficos de las Figuras 1 y 2 es posible advertir que en el modelo de Luhmann no aparece en ningún nivel el hombre como tal sino que se observa que este ha sido reemplazado por la principal operación que, según este autor, conforma el sistema: la comunicación. De esto se podría interpretar que la teoría de Luhmann está propuesta desde una visión de despersonalización, "con otras palabras, la propia teoría sistémica confiere una importancia inusitada a la capacidad de creación de imaginarios colectivos y estereotipos sociales, como formas de regulación (en el fondo control social) de los sistemas sociales" (Barraycoa, 2017), por lo que la comunicación dentro del sistema y con su entorno, estaría destinada a mantener el control de la sociedad mientras se autoorganiza y produce y reproduce de manera autopoiética para mantener su estructura en base a la diferenciación y la elección de las posibilidades contingentes que se generan. Lo que según la autora García expresa como: "donde las relaciones entre las partes y su influencia mutua son más importantes que la cantidad o el tamaño de las partes" (García, $\mathrm{s} / \mathrm{f})$, y se encuentra en coherencia con la despersonalización del hombre dentro del sistema al pretender Luhmann que las relaciones que se desarrollan en la comunicación del sistema poseen mayor importancia que las partes que lo conforman y pueden compararse "con el movimiento browniano, ya que se acentúa en él la disolución del lazo social” (Suárez, s/f).

Por otro lado, en cuanto sistema constituido por la comunicación que se establece entre los elementos que lo constituyen, es posible advertir que esta comunicación está soportada en base a un código comunicacional de tipo binario, el que también posibilita la operación de diferenciación "tendientes a la construcción de marcos de sentido y prácticas" (García, s/f) que conforman dicho código al interior de cada sistema, por lo que cada uno tendrá el suyo propio. Según esta teoría, para construirlo es preciso tener en cuenta "tres dimensiones la real - objetiva referida a objetos; la social a los sujetos; y la temporal al tiempo" (García, s/f), las cuales establecerán la complejidad.

\section{b) INFLUENCIA DE LA TEORÍA DE LUHMANN EN LA EDUCACIÓN INCLUSIVA}

Desde la teoría sistémica propuesta por Luhmann la educación es vista como un subsistema en el cual el código de comunicación que establece las relaciones entre los elementos, que autopoiéticamente se producen y reproducen al interior del mismo, posee una "binariedad [que] le permite al sistema enfrentar las modificaciones del entorno, su complejidad. Y si bien el código es una bifurcación de decisión, 
no es un criterio para la toma de decisiones ni una norma" (Ontiveros Quiroz, 1997), de lo que se entiende que estas decisiones son tomadas a partir de las posibilidades que existan en el código binario para regular el sistema y asegurar la conservación del mismo. De este modo se presupone que "si la realidad es contingente los fines no pueden quedar fijos" (Ontiveros Quiroz, 1997) y deben ser intercambiables en virtud de la prioridad que se le otorga a la funcionalidad del sistema y sus operaciones de comunicación por sobre la realidad.

En el mismo sentido es posible advertir que el sistema depende de la comunicación establecida entre sus partes a partir de un código binario que oscila entre posibilidades que pueden ser/ no ser y que frente "a una realidad contingente el fin pierde peso" (Ontiveros Quiroz, 1997) omitiéndose la visión teleológica de la educación y sustituyéndola por la funcionalidad de las operaciones de autopoiesis y autoorganización que permitan mantener al sistema en constante funcionamiento. Por lo tanto y "en este marco de teoría, la discusión sobre los fines del sistema educativo se vuelve no obsoleta sino inadmisible" (Ontiveros Quiroz, 1997) ya que el fin (del sistema) podría inscribirse en la decisión frente a las mejores posibilidades de conservar el funcionamiento del sistema sin tener en cuenta las partes que lo conforman sino las operaciones de comunicación que se establecen entre ellas para su subsistencia. Siendo así, "el esquema fines/medios encuentra aplicación en la escuela como sistema de organización" (Ontiveros Quiroz, 1997) dando prioridad de esta manera a la comunicación establecida entre los elementos de este sistema de organización y relegando la realidad de los mismos elementos que lo constituyen.

Ahora bien, este código binario que opera en base a las relaciones de comunicación establecidas entre sus partes

"obliga en la práctica a disponer de un conjunto de reglas decisionales que fijan las condiciones que permiten asociar cada valor con lo correcto o incorrecto. Tales reglas constituyen los programas. La distinción entre código y programa estructura la autopoiesis de los sistemas de funciones en forma inconfundible y la semántica que de allí resulta se distingue fundamentalmente de una tradición fincada en concepciones teleológicas, referidas a valores morales, a ideas de perfección u otros ideales" (Ontiveros Quiroz, 1997).

De esta manera, los programas definidos serán los que otorguen valor o sentido a los códigos que se empleen hacia el interior del sistema aceptando o rechazando la incorporación de nuevos elementos que se encuentren en el entorno o se produzcan dentro del mismo sistema en base a las reglas establecidas por la binariedad del código: correcto/incorrecto que orienta las operaciones del sistema educativo.

Así es posible advertir que el sistema educativo se conserva, es decir se produce y reproduce autopoiéticamente, en tanto la relación entre código y programas posean especificidad en su funcionalidad ya que, según aproximaciones a esta teoría, "si no existiera un instrumento de codificación binaria en el sistema educativo el hecho pedagógico se desintegraría en una red abierta de aprendizajes que se difundirían por todas partes y no constituiría una unidad discreta como sistema" (Ontiveros Quiroz, 1997) y dejaría de ser un sistema luhmanniano al confundirse con su entorno.

Sin embargo y teniendo en cuenta que la visión sistémica considera que un sistema se conforma de otros subsistemas y su entorno, en el caso particular del de la educación, existen otros subsistemas que tienen la misma funcionalidad que este y por lo que "la función de formación para el sistema educativo no es suficientemente específica" (Ontiveros Quiroz, 
1997) aunque posea un código y un programa destinado a operar en base a una comunicación establecida para la conservación del subsistema educativo. No obstante, mediante la clausura de operaciones logra establecer los límites de su funcionalidad y aunque estas funciones se realicen en el interior, "para el sistema educativo, su entorno, que incluye a los otros sistemas parciales, es educativamente competente" (Ontiveros Quiroz, 1997) porque opera en base al programa establecido de acuerdo al código binario que lo rige.

De este modo, "la identidad del sistema educativo no es una esencia expresada en sus fines; se entiende como una síntesis de posibilidades de ser de otra manera" (Ontiveros Quiroz, 1997) ya que la función del sistema educativo deviene en contingente por lo que puede ser/no ser de acuerdo a las relaciones que se establezcan entre los elementos que conforman el sistema. Así es posible comprender que "Luhmann propugna una educación sistémica sin inclusión del factor humano" (Suárez, s/f) con la carga que esto conlleva acerca de la omisión de la realidad de las partes que conforman al sistema, por lo que es posible afirmar que "la concepción sistémica aplicada a la educación ha servido para cosificar la educación" (Suárez, s/f) y despersonalizar el acto educativo transformándolo en el desempeño de una función, ignorando así a quienes lo integran tanto educadores como educandos y promocionando en este sentido una materialización de la educación que se traduce en la necesidad de acreditación de etapas de formación desprovistos de valores o ideales, aunque a este respecto, "la hipótesis es que el sistema busca enmascararlo recurriendo al establecimiento de fines para contar con un consenso que la selección no lograría" (Ontiveros Quiroz, 1997), ya que la operación decisoria, conforme al sentido otorgado al código binario de correcto/incorrecto, sería insuficiente para considerar los fines relacionados a valores o ideales que trasciendan la materialización o cosificación de la educación.

De este modo y notando la ausencia de un aspecto teleológico encontramos que en la peculiar percepción de Niklas Luhmann, al desarrollar su teoría de los sistemas, desde el aspecto antropológico de su visión, lleva a considerar sin duda que "se ha tratado al educando como una máquina trivial, y ahí radica precisamente el error. Las máquinas triviales se caracterizan porque reaccionan de una forma fija y constante a un determinado imput (sic) produciendo un output" (Suárez, s/f), lo que equivale a decir que si a una máquina trivial se la conecta se espera que funcione $y$ al momento de desconectarla se espera que deje de hacerlo evitando considerar que "las máquinas no triviales, por el contrario, no responden siempre de la misma manera, sino que lo hacen según su estado momentáneo" (García, s/f), característica que pertenece al ser humano, ya que no es posible regular sus outputs desde afuera y por el contrario las respuestas a cada input proviene desde su propio interior y de manera no previsible porque su propio interior no se rige por un código binario.

Es de considerar que esta situación se produce porque las máquinas no triviales

"son menos seguras que las triviales, pero más flexibles. Todos los sistemas psíquicos (niños y educandos...) son máquinas no triviales. Pero... pedagogos, maestros y educadores tratan al educando como una máquina trivial. Probablemente, dice Luhmann porque resultan más fáciles y cómodas de observar y evaluar" (García, s/f).

De lo anterior es posible comprender, nuevamente, que la primacía de la funcionalidad no permite la observación de la realidad para contemplar las particularidades de cada educando que se encuentra inserto en el sistema educativo. Sino que considerado como 
máquina trivial se lo evalúa y filtra de acuerdo a la binariedad característica del sistema educativo: correcto/incorrecto para determinar su pertenencia o no al mismo.

Entendiendo entonces que el sistema educativo actual responde al esquema de la teoría sistémica desarrollada por Niklas Luhmann, en la cual la conservación de la funcionalidad del sistema es condición necesaria y en la cual "la comunicación del poder no desaparece sino que deja de explicitarse" (Barraycoa, 2017), y por lo que se considera antropológicamente a quienes lo conforman como un todo perteneciente al sistema de relaciones de comunicación y que a su vez responden a un programa de sentido definido en los límites de contingencia que ofrece el código binario carente de visión teleológica, sería posible afirmar que la modalidad educativa de inclusión es una respuesta autopoiética que el mismo sistema educativo produce para mantener su funcionalidad y subsistencia.

\section{c) INTERPELACIÓN A LA TEORÍA}

\section{LUHMANNIANA: ¿Es posible contrarrestar el factor ateleológico y autorreferencial de la teoría de Luhman en la educación inclusiva?}

Teniendo en cuenta la reflexión de G. Suárez sobre la teoría luhmanniana acerca de que esta "refleja muy bien lo que hoy acontece a nivel social; es más, esclarece en forma muy certera la lógica subyacente en los diversos procesos sociales, que muchas veces escapan a nuestro entendimiento" (Suárez, s/f) y habiendo advertido la sustitución de una visión teleológica que considere al hombre como eje de la sociedad por la conservación del sistema mediante su funcionalidad, es posible dar continuidad a este pensamiento para indagar sobre los espacios en los cuales se podría considerar una intervención provocativa para recuperar el valor y sentido de las relaciones interpersonales.
En este sentido, podría considerarse principal la necesidad de una visión teleológica dentro del subsistema educativo y más aún en la modalidad de inclusión en tanto se pretende con esta la reinserción al sistema. De otro modo, es decir aplicando las mismas operaciones de funcionalidad de la teoría luhmanniana, se lograrán los mismos resultados y consecuentemente la modalidad de inclusión enfrentará, con el tiempo, las dificultades del sistema de educación común, tales como la deserción y la exclusión, lo que producirá el colapso de la modalidad inclusiva.

Considerando lo anterior y también teniendo en cuenta la reducida matrícula que actualmente posee la modalidad de inclusión, en relación a la educación común, podría ser este un intersticio que permita la reconstrucción de los vínculos interpersonales en torno a valores e ideales que guíen los fines y medios de la propuesta educativa para la inclusión de aquellos educandos que han sido excluidos del sistema educativo de modalidad común por diversos motivos.

A este respecto, la propuesta del personalismo, que parte de una visión antropológica opuesta a la teoría luhmanniana y enfatiza al hombre en su calidad de persona considerándola con su rasgo principal de integralidad, se constituye en un impulso capaz de otorgar una comprensión de la realidad actual del hombre en cuanto a su interioridad, recuperando el valor como persona y que "devolverá al mismo tiempo esa virtud interior que da autoridad, independencia y libertad respecto de las cosas" (Mounier, 1956).

Más aún, habiendo encontrado que la teoría sistémica es incapaz de reconocer la realidad y oscila entre las posibilidades previsibles de ser/no ser, sería pertinente reconocer a esta teoría funcionalista como no-funcional para la conservación de un sistema educativo que priorice a la persona, ya que subsiste en base a continuas modificaciones que responden a 
su característica de clausura de operaciones de código binario de correcto/incorrecto, incluido/excluido, aprobado/desaprobado. Un sistema con tal característica sobreviene nofuncional o impersonal porque la persona no es un abstracto programado sino que es una realidad concreta imprevisible, y "lo perverso es que queramos poner en ellos todo aquello que hemos expulsado de nosotros mismos para considerarnos inocentes y producir la dominación legítima" (Sai, 2017), situación que es notable en la modalidad educativa de inclusión, donde los excluidos vuelven a ser incluidos dentro del mismo sistema pero en un programa con reglas propias y que constantemente pone de relieve la condición previa.

Es por esto que "de su anti-humanismo, de este notable y radical rechazo al antropocentrismo en la comunicación, extraemos una filosofía del límite, sabiduría de la decadencia del hombre" (Sai, 2017) y deviene inminente una perspectiva que permita "retomar esta crítica 'materialista' y en librarla de las simplificaciones que le impusieron los primeros combates y de la supervivencia de un positivismo superado por la ciencia y la crítica" (Mounier, 1956), una crítica que pueda ir más allá de lo previsible y enfrente lo imprevisible del hombre como parte de la persona permitiéndole la búsqueda de un equilibrio entre su interior y su exterior.

\section{CONCLUSIÓN}

Revisar la teoría luhmanniana a la luz de una aproximación a la filosofía personalista encuentra un desafío entre opuestos que puede avanzar en equívocos toda vez que se entienda que Luhmann teorizó sobre la sociedad a partir de su experiencia como parte de la sociedad mientras Mounier se esforzó por recuperar el valor supremo del hombre después de la vivencia de los regímenes totalitarios que azotaron la sociedad en su época.
Aún así otro desafío encuentra este trabajo frente al interrogante planteado sobre la posibilidad de encontrar un espacio fértil dentro de la educación inclusiva para reflexionar sobre la propuesta de Mounier en medio de la teoría sistémica de Luhmann.

Seguramente habrá intersticios que así lo permitan y otros que rechacen la propuesta de volver a la consideración del hombre como persona más allá de estar rodeado y pertenecer a sistemas y entornos que a lo largo de su vida van cambiando. Con esto se pretende considerar una alternativa más dentro del sistema educativo que promueva la realización integral de la persona a partir del esclarecimiento que hace Luhmann en su teoría sobre el funcionamiento de la sociedad ya que no tenerlo en cuenta sería un error más grande que resistir la despersonalización que producen los sistemas.

\section{REFERENCIAS BIBLIOGRÁFICAS}

Barraycoa, J. (2017). Despersonalización y control social en la sociedad posmoderna. Espíritu - , LXVI (53), 87-106.

García, B. E. (s/f). La teoría de la Educación de Niklas Luhmann. Educación, Pensamiento Complejo y Desarrollo Institucional Integrado (págs. 1-20). Sala del CREDI de la OEI.

Mounier, E. (1956). ¿Qué es el personalismo? (E. Ruffo, Trad.) Buenos Aires: Ediciones Criterio.

Ontiveros Quiroz, S. J. (1997). Niklas Luhmann: una visión sistémica de lo educativo. Perfiles Educativos , XIX (78), 1-16.

Sai, L. F. (2017). El pensamiento antitotalitario de Luhmann como polémica contra el humanismo en cbernética. XII Jornadas de Sociología - Recorridos de una (in)disciplina - La sociología a sesenta años de la fundación de la carrera (págs. 1-10). Buenos Aires: Universidad de Buenos Aires. 
Suárez, G. (s/f). Una lectura alternativa

al estudio de la sociedad civil. La postura

sistémica de Niklas Luhmann. Algunas notas

sobre su impacto en el sub-sistema educativo.

$s / d . s / d$ 